\title{
Topology reaches higher spheres
}

\author{
Topology is everywhere. Recent predictions for and realizations of higher-order topological insulators are a case in
}

point.

$\mathrm{D}$ uncan Haldane observed, pointedly, that "just knowing the correct laws of quantum mechanics does not mean that we understand all the strange phenomena that it allows." So he spoke in his banquet speech, just having been awarded the 2016 Nobel Prize in Physics, together with David Thouless and Michael Kosterlitz. The work of the trio, and others, on topological phase transitions and topological phases has flung open the gate to the discovery of countless mind-boggling properties and behaviours of matter - "'really cool things' that had never been guessed at before", as Haldane put it.

In the two years since Haldane's speech, we have seen many more 'really cool' developments: a case in point being higherorder topological insulators, which feature prominently in the current issue of Nature Physics.

Topological insulators (TIs) are, in a nutshell, systems with an insulating bulk and topologically protected (and therefore robust) excitations at their boundary. 'Conventional' two-dimensional TIs exhibit such fractionalization on their edges, three-dimensional ones at their surface. In contrast, higher-order topological insulators - or, of course, HOTIs - are topologically protected at the boundary of the boundaries, further reducing the dimensionality.
Following the first proposals ${ }^{1}$ for such exotic states, experimental realizations of twodimensional electric quadrupole insulators with protected corner states were soon reported. Intriguingly, these demonstrations came on a variety of classical platforms: photonic crystals $^{2}$, mechanical pendula ${ }^{3}$, microwave resonators ${ }^{4}$ and, as Stefan Imhof and colleagues ${ }^{5}$ report in this issue, electrical circuits.

Whereas the ability to create topologically non-trivial phases in such metamaterials is in itself no longer a surprise, the fact that electric multipole insulators have first been realized in analogue systems, rather than in 'natural' electronic systems, is noteworthy. There could even be an opportunity for science outreach, especially in the context of the work of Imhof et al., who use (as a few other groups do) RLC circuits to emulate the behaviour of topological matter. As Ling $\mathrm{Lu}^{6}$ writes in his accompanying News \& Views, the ability to explore one of the most active areas of physics using a set of off-the-shelf electronics components should be a blessing for students and teachers alike. No more explaining TIs with coffee mugs morphing into doughnuts.

But the story does not end with these analogue experiments. The concept of electric multipole insulators led to a broader theory of HOTIs, generalizing the topological bulk-boundary correspondence ${ }^{7}$. In this issue, Frank Schindler and colleagues ${ }^{8}$ report that bismuth - a material that has already presented us with so many surprises ${ }^{9}-$ is a realization of a HOTI. And bismuth is unlikely to remain alone. In a series of very recent computational studies ${ }^{10}$, thousands of materials have been predicted to possess topological electronic properties, rather than the few hundred that are currently known, among them a substantial number of HOTIs. We're only just scratching the surface, with further 'strange phenomena' undoubtedly waiting underneath.

Published online: 4 September 2018 https://doi.org/10.1038/s41567-018-0288-4

\section{References}

1. Benalcazar, W. A., Bernevig, B. A. \& Hughes, T. L. Science 357, 61-66 (2017).

2. Noh, J. et al. Nat. Photon. 12, 408-415 (2018).

3. Serra-Garcia, M. et al. Nature 555, 342-345 (2018).

4. Peterson, C. W., Benalcazar, W. A., Hughes, T. L. \& Bahl, G. Nature 555, 346-350 (2018).

5. Imhof, S. et al. Nat. Phys. https://doi.org/10.1038/s41567-018 0246-1 (2018).

6. Lu, L. Nat. Phys. https://doi.org/10.1038/s41567-018-0235-4 (2018).

7. Schindler, F. et al. Sci. Adv. 4, eaat0346 (2018).

8. Schindler, F. et al. Nat. Phys. https://doi.org/10.1038/s41567-0180224-7 (2018).

9. Ast, C. R. Nat. Phys. https://doi.org/10.1038/s41567-018-0258-x (2018).

10. Gibney, E. Nature 560, 151-152 (2018).

\section{Fields of joy}

\author{
In praise of the Fields Medal.
}

$\mathrm{T}$ he announcement of the 2018 Fields Medal winners, made last month at the opening of the International Congress of Mathematicians held in Rio de Janeiro, Brazil, was greeted with widespread acclaim well beyond the mathematics community.

Of the four winners, the work of Alessio Figalli on optimal transport, which seeks the most efficient way to distribute goods on a network, is probably the most straightforward to connect to the real world. The recognized contributions of the other three awardees, Caucher Birkar, Peter Scholze and Akshay Venkatesh, are on more abstract topics concerning algebraic varieties, $p$-adic fields and number theory, respectively - humbling subjects even for the most theoretically inclined of physicists.

Although often referred to as the Nobel Prize of mathematics, the Fields Medal is in fact very different in terms of its procedures, criteria, remuneration and much else. Notably, the Nobel is typically given to senior figures, often decades after the contribution being honoured. By contrast, Fields medallists must all be under 40, an age at which, in most sciences, a promising career would just be taking off.
Indeed, the most famous instruction left by the prize's main proponent, John Charles Fields, was that the awards should be both "in recognition of work already done" and "an encouragement for further achievement".

So let us celebrate the work of these brilliant young mathematicians. And let us encourage them to further achievements that will doubtless spill into physics as well.

Published online: 4 September 2018 https://doi.org/10.1038/s41567-018-0289-3 